\title{
Notifications of Public Health Events under the International Health Regulations - 5 Year U.S. Experience
}

\author{
Katrin S. Kohl*1, Cody Thornton ${ }^{2}$, Jose Fernandez ${ }^{2}$, Nicki Pesik ${ }^{1}$, Francisco Alvarado- \\ Ramy ${ }^{1}$, Martin Cetron ${ }^{1}$ and Ray Arthur ${ }^{1}$ \\ ${ }^{1}$ Centers for Disease Control and Prevention, Atlanta, GA, USA; ${ }^{2}$ Office of the Assistant Secretary for Preparedness \& Response, \\ Washington, DC, USA
}

\section{Objective \\ We looked at the public health impact in the US of the notification requirement of potential public health emergencies of international concern (PHEIC) under the 2005 International Health Regulations (IHR) to the World Health Organization (WHO). \\ Introduction \\ All WHO member states are signatories of the IHR (1) to improve global health security. Objectives are to improve countries' capacities to detect, notify and respond to PHEICs. Rapid and transparent infor- mation sharing are critical for global coordination and rapid response to minimize an event's impact on the public. The IHR require coun- tries to establish a point of contact, or National Focal Point (NFP), re- sponsible for notifying WHO of potential PHEICs and responding to requests of information. Potential PHEICs are assessed by the country and re-assessed by WHO, which posts its assessment together with information on response measures on a secure IHR Event information Site (EIS) accessible by all NFPs.}

\section{Methods}

We reviewed two types of IHR notifications: 1) notifications by the US NFP to WHO of potential PHEICs as they were submitted to WHO and as they were posted by WHO on EIS; 2) notifications by other countries' NFPs to the US NFP about health events in those countries that directly impact the US. Finally, we reviewed available information on public health actions taken by US public health agencies or other countries' agencies in response to reported events.

\section{Results}

The US NFP reported 59 potential PHEICs to WHO (from 8/2007 to $7 / 2013$ ); 32 were for influenza (2). WHO has posted 33 separate events for the US on EIS, which lists 274 events from 110 countries. The discrepancy in the number of events reported and posted is likely due to groupings of several reported events or disparate risk assessment by WHO. 2009 pandemic H1N1 influenza was first reported by the US NFP on April 17, 2009, and determined to constitute a PHEIC on April 25, 2009. It remains the only PHEIC to date. Direct interactions with WHO on potential PHEICs have resulted in WHO's assistance with international contact tracing and sharing of survey or communication materials. The establishment of NFPs has led to regular exchanges of health information between NFPs to enable the receiving country to take appropriate public health action (e.g., providing prophylaxis for meningococcal disease). Most notifications by other countries' NFPs to the US were for travelers with tuberculosis or measles. When the information is transmitted to local public health, exposed travelers may be contacted. Sharing results of contact investigations by local public health to federal authorities is voluntary and often not done. There is equally no systematic information available on actions taken by countries in response to postings of potential
PHEICs, with the exception of events in which WHO provided direct assistance.

\section{Conclusions}

The implementation of pathways and protocols for assessing and reporting public health threats to WHO under the IHR has resulted in timely notifications. While not all notifiable events are being reported and not all reported events are being posted, the framework of reporting enables a level of transparency and information that might be useful to countries not well connected in other global health security settings. However, information on what countries do in response to postings of events on the IHR intranet site is lacking. At the same time, bilateral or direct exchange with WHO has yielded actionable information and enabled coordinated responses. Lack of baseline information of bilateral exchange on exposure risks makes it impossible to know if the existence of NFPs increased such exchange, or domestically, if it prevented disease. However, we know from experience that it made such notifications easier. Further analysis of the impact of notifications is needed.

\section{Keywords}

global health; risk assessment; event notification; international health regulations

\section{References}

1. World Health Organization. International Health Regulations 2005,2nd ed. Geneva: The Organization; 2008.http://whqlibdoc.who.int/publications/2008/9789241580410_eng.pdf.

2. Kohl KS, Arthur RA, O’Connor R, Fernandez J. Assessment of public health events through international health regulations, United States, 2007-2011. Emerg Infect Dis 2012 Jul. http://dx.doi.org/10.3201/ eid1807.120231.

\section{*Katrin S. Kohl}

E-mail: kkohl@cdc.gov 